Urol. int. 1965;19:1

\title{
Symposium I: Endocrinosurgery in the Cancer of the
}

\section{Prostate}

Endocrinochirurgia del cancro della prostata

Endocrinochirurgie dans le cancer de la prostate

Endokrinochirurgie beim Prostatakrebs

Endocrinocirugia en el cancer de la prostata

Chairman: Prof. M. Sorrentino

INDEX

Gil Vernet, S. (Barcelona): Correlaciones entre glandulas endocrinas y

prostata

3

Jönsson, G. (Lund): Estradurin ${ }^{\circledR}$ (Polyoestradiol phosphate) in the treatment of prostatic carcinoma. A clinical and steroid metabolic study

Pavone, M. (Palermo): L'orchiectomia nella terapia del cancro della prostata

Defoort, R. (Gand): La surrénalectomie bilatérale dans le traitement du

cancer de la prostate 30

Bracci, U. (Roma): Considerazioni critiche sulla surrenectomia chirurgica 41

Sorrentino, F. (Napoli): L'alcoolizzazione delГipofisi nella terapia del

cancro della prostata 47

Greco, T. (Firenze): L'alcoolizzazione della ipofisi. Vantaggi di questometodo nel trattamento dei tumori maligni avanzati e delle loro ripro-duzioni 54

Belt, E. (Los Angeles): Cancer of the Prostate, Total Perineal Prostatectomy

Cure and Survival Rates 58

Couvelaire, R. (Paris): Sur le traitement chirurgical du cancer prostatique 63

S. Sorrentino

21. (Napoli): Conclusioni

67

2 Urol. int.

Vol. 19

No. 1 (1965) 